\title{
On the approximation of the canard explosion point in singularly perturbed systems without an explicit small parameter
}

\author{
Brøns, Morten; Kristiansen, Kristian Uldall
}

Published in:

Dynamical Systems

Link to article, DOI:

$10.1080 / 14689367.2017 .1313390$

Publication date:

2017

Document Version

Early version, also known as pre-print

Link back to DTU Orbit

Citation (APA):

Brøns, M., \& Kristiansen, K. U. (2017). On the approximation of the canard explosion point in singularly perturbed systems without an explicit small parameter. Dynamical Systems, 33(1), 136-158.

https://doi.org/10.1080/14689367.2017.1313390

\section{General rights}

Copyright and moral rights for the publications made accessible in the public portal are retained by the authors and/or other copyright owners and it is a condition of accessing publications that users recognise and abide by the legal requirements associated with these rights.

- Users may download and print one copy of any publication from the public portal for the purpose of private study or research.

- You may not further distribute the material or use it for any profit-making activity or commercial gain

- You may freely distribute the URL identifying the publication in the public portal

If you believe that this document breaches copyright please contact us providing details, and we will remove access to the work immediately and investigate your claim. 


\title{
On the approximation of the canard explosion point in epsilon-free systems
}

\author{
M. Brøns and K. Uldall Kristiansen \\ Department of Applied Mathematics and Computer Science \\ Technical University of Denmark \\ 2800 Kongens Lyngby \\ Denmark
}

January 2, 2018

\begin{abstract}
A canard explosion is the dramatic change of period and amplitude of a limit cycle of a system of non-linear ODEs in a very narrow interval of the bifurcation parameter. It occurs in slow-fast systems and is well understood in singular perturbation problems where a small parameter epsilon defines the time scale separation. We present an iterative algorithm for the determination of the canard explosion point which can be applied for a general slow-fast system without an explicit small parameter. We also present assumptions under which the algorithm gives accurate estimates of the canard explosion point. Finally, we apply the algorithm to the van der Pol equations and a Templator model for a self-replicating system with no explicit small parameter and obtain very good agreement with results from numerical simulations.
\end{abstract}

\section{Introduction}

We consider singular perturbation problems of the form

$$
\begin{aligned}
& \dot{x}=F(x, y, z, \epsilon), \\
& \dot{y}=G(x, y, z, \epsilon),
\end{aligned}
$$

where $\epsilon$ is small parameter and $z$ is a bifurcation parameter. All vector fields are assumed to be analytic in their arguments. In the singular limit $\epsilon=0$ the system is assumed to have a critical manifold, that is, a manifold of critical points. We restrict our attention to planar systems with $x \in \mathbb{R}$ and $y \in \mathbb{R}$ such that the critical manifold is a curve. The parameter $\epsilon$ defines a time-scale separation, and for singular perturbation problems in the special form

$$
\begin{aligned}
& \dot{x}=F(x, y, z, \epsilon), \\
& \dot{y}=\epsilon H(x, y, z, \epsilon),
\end{aligned}
$$

the state variables $x$ and $y$ can be identified as the fast and the slow variable, respectively. For the system (2) the critical manifold is defined by $F(x, y, z, 0)=0$, and it follows from Fenichel theory $[7,9]$ that, under certain regularity conditions, there exists a slow invariant manifold $\epsilon$-close to the critical manifold. For simplicity, we will suppress the explicit dependence of $F$ and $H$ on $\epsilon$ in the system (2) from now on. 
Canard explosion. The canard explosion is the dramatic change of amplitude and period of a limit cycle born in a Hopf bifurcation in a very narrow parameter interval. The phenomenon is well understood in singular perturbation problems of the form $(2)[1,17,11,3]$. Canards are solutions which are $\epsilon$-close to a critical manifold on the form $\eta_{0}=\eta_{0}(y, z)$ defined from $F\left(\eta_{0}(y, z), y, z\right)=0$ both on an attracting part where $\partial_{x} F<0$ and a repelling part where $\partial_{x} F>0$. Thus, canards are slow manifolds with both an attracting and a repelling part. They only exist for a narrow range (of order $e^{-c / \epsilon}$ ) of the parameter $z$, and the canard explosion occurs when a segment of a limit cycle is a canard. A unique asymptotic expansion of a parameter value $z$ where the canard occurs, the canard point, and an expansion of the corresponding (maximal) canard can readily be obtained.

However, canard explosions are also observed in slow-fast systems where there is no explicit small parameter that separates the timescales,

$$
\begin{aligned}
& \dot{x}=F(x, y, z), \\
& \dot{y}=H(x, y, z),
\end{aligned}
$$

and where existing singular perturbation theory cannot be applied directly. An example is the Templator model which we consider in Section 3. In [5] a modification of the iterative method of Fraser and Roussel $[8,14]$, devised to construct regular slow manifolds, was proposed to determine a canard point for a general system of the form (3). The method was successfully applied to the van der Pol equations where it was shown that the first terms in the asymptotic expansion of the canard point are determined correctly, and on a Templator model for a self-replicating system with no explicit $\epsilon$ where canard explosions have been found numerically.

In this paper, we will consider a modified version of this method. Being based on a linearization, the method is explicit, in contrast to the original one. Furthermore, we present explicit nondegeneracy conditions as well as "smallness conditions" and show how they guarantee accurate approximations for the canard solution and the canard explosion point for the system (3). We illustrate the method with examples.

Notation. All norms will be denoted by $|\cdot|$. Superscripts with $n \in \mathbb{N}_{0}$ will be used to denote partial sums such as:

$$
\eta^{n}=\sum_{i=0}^{n} \eta_{i}, \quad n \geq 0,
$$

with each of the terms in the sum being enumerated through subscripts. Following this convention means that $\eta^{0}=\eta_{0}$. We will in this paper suppose that the vector-field is analytic. In particular we will consider complex sets of the form $x \in(a, b)+i \nu$ and $y \in(c, d)+i \sigma$. Here we by $(a, b)+i \nu \subset \mathbb{C}$ and $(c, d)+i \sigma \subset \mathbb{C}$ denote the complex $\nu$ and $\sigma$-neighborhoods of the real intervals $(a, b)$ resp. $(c, d)$. We will then use $|h|_{\nu}$ to denote the sup-norm of an analytic function $h$ over the domain $(a, b)+i \nu$. This representation gives the following compact form of Cauchy's estimate:

Lemma 1 Let $h=h(x)$ be analytic in $x \in(a, b)+i \nu$. Then

$$
\left|h^{\prime}\right|_{\nu-\xi} \leq \frac{|h|_{\nu}}{\xi}, \quad 0<\xi \leq \nu .
$$

Proof See [15, Theorem 10.26].

The iterative method of Fraser and Roussel. A slow manifold for Eqns. (2) of the form $x=\eta(y, z)$ fulfills the invariance equation

$$
-\epsilon \partial_{y} \eta H(\eta, y, z)+F(\eta, y, z)=0,
$$


obtained by eliminating time. Fraser and Roussel $[8,14]$ developed an iterative method for the approximation of slow manifolds from the scheme

$$
-\epsilon \partial_{y} \eta^{n-1} H\left(\eta^{n}, y, z\right)+F\left(\eta^{n}, y, z\right)=0
$$

starting from the critical manifold $\eta^{0}=\eta_{0}(y, z)$. The function $\eta=\eta^{n}$ satisfies (5) up to the error

$$
-\epsilon \partial_{y} \eta_{n} H\left(\eta^{n}, y, z\right)
$$

using the notation (4). In [16] it was shown using Cauchy estimates to control (7) that this procedure, even for an arbitrary number of slow and fast variables, leads to slow manifolds exponentially close $\left(\mathcal{O}\left(e^{-c / \epsilon}\right)\right)$ to invariance. The equation (6) is non-linear in $\eta^{n}$, but Neishtadt [13] showed that the same convergence is obtained if $F$ and $H$ in (6) are linearized with respect to $x$ at $\eta^{n-1}$ such that the equation becomes linear in $\eta_{n}=\eta^{n}-\eta^{n-1}$.

A crucial assumption for the success of these methods is that $\left|\partial_{x} F\right| \gg \epsilon$. This is violated near fold points of the critical manifold where $\partial_{x} F=0$, and this is where canards may occur. To study canards in systems with fold points one instead makes use of the fact that $\partial_{y} F \neq 0$ and solve $F(x, y, z)=0$ for $y=\zeta_{0}(x, z)$. A fold point $\left(x_{0}, \zeta_{0}\left(x_{0}, \mu_{0}\right)\right)$ is then characterized by $\partial_{x} \zeta_{0}\left(x_{0}, \mu_{0}\right)=0$ for some parameter value $z=\mu_{0}$. For a slow manifold $y=\zeta(x, z)$ the invariance equation becomes

$$
-\partial_{x} \zeta F(x, \zeta, z)+\epsilon H(x, \zeta, z)=0
$$

and following Fraser and Roussel it is proposed in [5] to solve this iteratively from

$$
-\partial_{x} \zeta^{n-1} F\left(x, \zeta^{n}, z\right)+\epsilon H\left(x, \zeta^{n}, z\right)=0
$$

starting with the critical manifold $\zeta^{0}=\zeta_{0}$. A solution $\zeta^{n}(x, z)$ of $(9)$ will generally have a singularity close to the fold, but this may be canceled by an appropriate choice of $z=\mu^{n}$. Then $\zeta^{n}$ is welldefined at the fold, and hence represents a canard. Thus, this procedure yields a sequence $\mu^{n}$ of approximations to the canard point as well as approximations $y=\zeta^{n}\left(x, \mu^{n}\right)$ of the corresponding canard. In the present paper we follow Neishtadt again, and linearize Eqn. (9) at $\zeta^{n-1}$ such that it can be solved explicitly for $\zeta^{n}$. A precise formulation of this procedure requires some notation which we turn to now; the complete algorithm is described as Algorithm 1 below.

\section{The modified Fraser-Roussel algorithm for canards}

Consider the system (1) and let

$$
V(x, y, z, \epsilon)=\left(\begin{array}{l}
F(x, y, z, \epsilon) \\
G(x, y, z, \epsilon)
\end{array}\right)
$$

denote the vector-field. A critical manifold for (1) is a smooth curve of fixed points $V(x, y, z, 0)=0$ within the $(x, y)$-plane for $(1)_{\epsilon=0}$. We shall assume that this manifold can be parametrized by $x$ so that $y=\zeta_{0}(x, z)$. We then have

Lemma 2 The critical manifold is normally hyperbolic at $\left(x_{0}, \zeta_{0}\left(x_{0}, z\right)\right)$ if and only if

$$
\partial_{y} V \cdot\left(-\partial_{x} \zeta_{0}, 1\right) \neq 0
$$


Proof The layer equations of (1) are

$$
\left(\begin{array}{c}
\dot{x} \\
\dot{y}
\end{array}\right)=V(x, y, z, 0)
$$

where $\dot{x}$ and $\dot{y}$ in general are both non-zero. An equilibrium $\left(x_{0}, \zeta_{0}\left(x_{0}, z\right)\right)$ is then normally hyperbolic if the linearization, described by the Jacobian:

$$
D V=\left(\begin{array}{ll}
\partial_{x} V & \partial_{y} V
\end{array}\right),
$$

only has one zero eigenvalue. Since $V\left(x, \zeta_{0}(x, z), z, 0\right)=0$ we have by implicit differentiation that

$$
\partial_{x} V=-\partial_{y} V \partial_{x} \zeta_{0}
$$

Therefore we can write $D V$ as

$$
D V=\left(\begin{array}{ll}
-\partial_{y} V \partial_{x} \zeta_{0} & \partial_{y} V
\end{array}\right)
$$

The eigenvalues of $D V$ are then directly obtained:

$$
0 \text { and } \partial_{y} V \cdot\left(-\partial_{x} \zeta_{0}, 1\right)
$$

and the result therefore follows.

Following Lemma 2, the critical manifold $y=\zeta_{0}(x, z)$ loses normal hyperbolicity at point $\left(x_{0}, \zeta_{0}\left(x_{0}, z\right)\right)$ where

$$
\partial_{y} V \cdot\left(-\partial_{x} \zeta_{0}, 1\right)=0
$$

Generically (see (A1) below) such a point is a fold point.

Remark 1 Geometrically, (10) means that the critical fiber of $\left(x_{0}, \zeta_{0}\left(x_{0}, z\right)\right)$ is tangent to the graph $y=\zeta_{0}(x, z)$ at this point.

One of the main aims of this paper is to present a method that applies to systems of the form (3) without an explicit $\epsilon$. To this end, we define a manifold $y=\zeta_{0}(x, z)$ from

$$
F\left(x, \zeta_{0}(x, z), z\right)=0 .
$$

Were there an $\epsilon$ multiplying $H$, as in (2), this would be the critical manifold for $\epsilon=0$ which can be used be used as a starting point for a Fraser-Roussel iteration as described in $\S 1$. The precise assumptions needed for this to work in the $\epsilon$-free setting are given in (A1) and (A2) below.

To continue, let $\left(x_{0}, \mu_{0}\right)$, be fixed, their values to be determined later in (A1), and introduce $y_{0}$ and $z_{0}$ by

$$
\begin{aligned}
& y=\zeta_{0}(x, z)+y_{0}, \\
& z=\mu_{0}+z_{0} .
\end{aligned}
$$

This leads to the following extended system obtained from (2):

$$
\begin{aligned}
\dot{x} & =F_{0}\left(x, y_{0}, z_{0}\right)=f_{0}(x)+F_{0 y}\left(x, y_{0}, z_{0}\right) y_{0}+F_{0 z}\left(x, y_{0}, z_{0}\right) z_{0}, \\
\dot{y}_{0} & =e_{0}(x)+\left(\Phi(x)+b_{0}(x)\right) z_{0}+T_{0}\left(x, z_{0}\right)+\left(\Lambda(x)+a_{0}\left(x, z_{0}\right)\right) y_{0}+R_{0}\left(x, y_{0}, z_{0}\right), \\
\dot{z}_{0} & =0
\end{aligned}
$$


where

$$
\begin{aligned}
F_{0 y}\left(x, y_{0}, z_{0}\right) & =\left(\text { using that } F\left(x, \zeta_{0}\left(x, \mu_{0}\right), \mu_{0}\right)=0\right) \\
& =\int_{0}^{1} \partial_{y} F\left(x, \zeta_{0}\left(x, \mu_{0}\right)+s y_{0}, \mu_{0}+s z_{0}\right) d s, \\
F_{0 z}\left(x, y_{0}, z_{0}\right) & =\int_{0}^{1} \partial_{z} F\left(x, \zeta_{0}\left(x, \mu_{0}\right)+s y_{0}, \mu_{0}+s z_{0}\right) d s, \\
e_{0}(x) & =G\left(x, \zeta_{0}\left(x, \mu_{0}\right), \mu_{0}\right), \\
\Phi(x) & =-\partial_{x} \zeta_{0}\left(x, \mu_{0}\right) \partial_{z} F\left(x, \zeta_{0}\left(x, \mu_{0}\right), \mu_{0}\right)+\partial_{z} G\left(x, \zeta_{0}\left(x, \mu_{0}\right), \mu_{0}\right), \\
\Lambda(x) & =-\partial_{x} \zeta_{0} \partial_{y} F\left(x, \zeta_{0}\left(x, \mu_{0}\right), \mu_{0}\right)+\partial_{y} G\left(x, \zeta_{0}\left(x, \mu_{0}\right), \mu_{0}\right),
\end{aligned}
$$

and $T_{0}=\mathcal{O}\left(z_{0}^{2}\right), a_{0}=\mathcal{O}\left(z_{0}\right)$, and $R_{0}=\mathcal{O}\left(y_{0}^{2}\right)$. Here we have just Taylor-expanded the right hands sides about $\left(y_{0}, z_{0}\right)=(0,0)$. For later convenience we have also introduced $f_{0} \equiv 0$ and $b_{0} \equiv 0$. The subscripts on $y_{0}, z_{0}$ and the functions $f_{0}, \ldots, b_{0}, \ldots, R_{0}$ are used to indicate that they later will be part of an iteration. The functions $\Lambda$ and $\Phi$ will not be updated.

The function $\Lambda=\Lambda(x)$ in (14) is precisely $\partial_{y} V \cdot\left(-\partial_{x} \zeta_{0}, 1\right)$ which according to (10) vanishes at a fold point $\left(x_{0}, \zeta_{0}\left(x_{0}, \mu_{0}\right)\right)$ at a given parameter value $z=\mu_{0}$. Therefore we will assume that there is such a point where $\Lambda$ vanishes. This only gives one condition on the pair $\left(x_{0}, \mu_{0}\right)$. For the possibility of having a canard solution we need further conditions. These are contained in the following assumptions:

(A1) The pair $\left(x_{0}, \mu_{0}\right)$ is so that $\Lambda$ (14) and $e_{0}$ (12) vanish:

$$
\begin{gathered}
-\partial_{x} \zeta_{0}\left(x_{0}, \mu_{0}\right) \partial_{y} F\left(x, \zeta_{0}\left(x_{0}, \mu_{0}\right), \mu_{0}\right)+\partial_{y} G\left(x_{0}, \zeta_{0}\left(x, \mu_{0}\right), \mu_{0}\right)=0, \\
G\left(x_{0}, \zeta_{0}\left(x_{0}, \mu_{0}\right), \mu_{0}\right)=0 .
\end{gathered}
$$

Given that $\Lambda\left(x_{0}\right)=0$ and $e_{0}\left(x_{0}\right)=0$ we can define $\tilde{\Lambda}$ and $\tilde{e}_{0}$ by

$$
\begin{aligned}
\tilde{\Lambda}(x) & =\int_{0}^{1} \partial_{x} \Lambda\left(x_{0}+s\left(x-x_{0}\right)\right) d s \\
\text { respectively } & \\
\tilde{e}_{0}(x) & =\int_{0}^{1} \partial_{x} e_{0}\left(x_{0}+s\left(x-x_{0}\right)\right) d s
\end{aligned}
$$

so that $\Lambda(x)=\left(x-x_{0}\right) \tilde{\Lambda}(x)$ and $e_{0}(x)=\left(x-x_{0}\right) \tilde{e}_{0}(x)$. We further assume:

(A2) The following non-degeneracy and "smallness" conditions hold true: Let

$$
\begin{aligned}
\tilde{\delta}_{0} & \equiv\left|\tilde{e}_{0}\right|_{\nu_{0}}, \\
K & \equiv\left|\tilde{\Lambda}^{-1}\right|_{\nu_{0}} .
\end{aligned}
$$

Then there exist $\epsilon \ll 1$ so that

$$
\tilde{\delta}_{0} \leq K^{-1} \epsilon,
$$

and

$$
\left|F_{0 y}\right|_{\nu_{0}},\left|F_{0 z}\right|_{\nu_{0}},\left|T_{0}\right|_{\nu_{0}},\left|R_{0}\right|_{\nu_{0}} \ll K^{-1} \epsilon^{-1} .
$$

Furthermore, either of the following conditions hold true: 
(a)

$$
\left|\Phi\left(x_{0}\right)\right|^{-1} \ll K^{-1} \epsilon^{-1}
$$

and

$$
|\Phi|_{\nu_{0}} \ll K^{-1} \epsilon^{-1}
$$

(b)

$$
\left|\Phi\left(x_{0}\right)\right|^{-1} \leq K^{-1} \epsilon^{-1}
$$

and

$$
|\Phi|_{\nu_{0}} \leq K^{-1} \epsilon
$$

Remark 2 We include case (b) in (A2) to cover the case where $G=\epsilon H$ is small.

The reason for supposing analyticity is that we in this case can present what we believe are optimal exponential estimates. However, we will not need any smoothness assumptions on how $\epsilon$ enters beyond condition (A1) and (A2). In particular, the analysis of our method is not based on asymptotic expansions in $\epsilon$.

The function $\tilde{e}_{0}$ in (15) is the obstacle to invariance of $y_{0}=0, z_{0}=0$ : If $\tilde{e}_{0} \equiv 0$ then $y_{0}=$ $0, z_{0}=0$ corresponds to a canard solution. We have therefore introduced $\tilde{\delta}_{0}$ in (16) as the error $\tilde{\delta}_{0}=\left|\tilde{e}_{0}\right|_{\nu_{0}}=\mathcal{O}(\epsilon)$. The system (11) is our normal form. Our algorithm will be based upon applying transformations, affine in $y_{0}$ and $z_{0}$, to (11) that seek to diminish the error $\tilde{e}_{0}$. These transformations directly lead to a simple algorithm similar to that presented in [5] that we present in Algorithm 1.

Remark 3 Suppose that $G=\epsilon H$ is truly small and the slow and fast variables have been properly identified. Then there are known sufficient conditions for a canard explosion [3, 10]:

(B1) There exists a pair $\left(\tilde{x}_{0}, \tilde{\mu}_{0}\right)$ so that

$$
\partial_{x} \zeta_{0}\left(\tilde{x}_{0}, \tilde{\mu}_{0}\right)=0, \quad H\left(\tilde{x}_{0}, \zeta_{0}\left(\tilde{x}_{0}, \tilde{\mu}_{0}\right), \tilde{\mu}_{0}\right)=0 .
$$

(B2) The following non-degeneracy conditions hold true:

$$
\begin{array}{r}
\partial_{y} F\left(\tilde{x}_{0}, \zeta_{0}\left(\tilde{x}_{0}, \tilde{\mu}_{0}\right), \tilde{\mu}_{0}\right) \neq 0, \partial_{x}^{2} F\left(\tilde{x}_{0}, \zeta_{0}\left(\tilde{x}_{0}, \tilde{\mu}_{0}\right), \tilde{\mu}_{0}\right) \neq 0, \\
\partial_{z} H\left(\tilde{x}_{0}, \zeta\left(\tilde{x}_{0}, \tilde{\mu}_{0}\right), \tilde{\mu}_{0}\right) \neq 0, \partial_{x} H\left(\tilde{x}_{0}, \zeta_{0}\left(\tilde{x}_{0}, \tilde{\mu}_{0}\right), \tilde{\mu}_{0}\right) \neq 0,
\end{array}
$$

Our condition (A1) replaces (B1). They are equivalent when $\partial_{x}^{2} F\left(x_{0}, \zeta_{0}\left(x_{0}, \mu_{0}\right), \mu_{0}\right) \neq 0$ by the implicit function theorem. Similarly, using (A1), it follows that the first three inequalities (B2) are equivalent to those in (A2), case (b), for $\epsilon$ sufficiently small. In condition (A2), however, we do not require $\partial_{x} H\left(\tilde{x}_{0}, \zeta_{0}\left(\tilde{x}_{0}, \tilde{\mu}_{0}\right), \tilde{\mu}_{0}\right) \neq 0$. This condition is included in (B2) because it guarantees that the nullclines of $x$ and $y$ are transverse at the fold point and that the equilibrium undergoes a Hopf bifurcation. It is the associated limit cycles that undergo rapid amplitude growth in the canard explosion. In agreement with [3], our algorithm and main result (Theorem 1 below) still apply without the need of this assumption but the results may have little dynamical significance. There is a well-known connection between the first Liapounov coefficient, the Hopf point and the canard point to lowest order in $\epsilon[11,3]$. This was exploited in [12] as a numerical tool to estimate canard explosion points. 
Remark 4 Suppose again that the slow and fast variables have been properly identified. Then one can actually replace $\Lambda$ in (14) by $-\partial_{x} \zeta_{0} \partial_{y} F$ and ignore the small term $\partial_{y}(\epsilon H)$. Our main result still applies as the term ignored can be collected into $a_{0}$. Indeed, the iterative lemma, Lemma 3 , that is the basis of our proof of the main theorem, just assumes that $a_{0}(x, 0)$ is bounded from above by $c \epsilon$, see (41) below, for some $c$ sufficiently large. However, in the general case, this term cannot be ignored. See also section 3.4 where we apply our algorithm to the Templator model.

Remark 5 As described in Remark 4, the result of the paper still applies if $\left|a_{0}(x, 0)\right|_{\nu_{0}} \ll|\tilde{\Lambda}|_{\nu_{0}}$. Similarly, we can also allow for $\left|f_{0}\right|_{\nu_{0}} \ll|\tilde{\Lambda}|_{\nu_{0}}$ and $\left|b_{0}\right|_{\nu_{0}} \ll|\Phi|_{\nu_{0}}$. The iterative lemma, Lemma 3 , still applies (see also (41) below).

The modified iterative method for the computation of canard explosion. We are looking for a canard solution through a graph $y=\zeta(x)$ for a value $\mu$ of the parameter $z$. The invariance of the graph gives the equation (8) for $\zeta=\zeta(x)$, which following Neishtadt [13] we wish to approach iteratively starting from $y=\zeta_{0}\left(x, \mu_{0}\right), z=\mu_{0}$ and continue with solving the linear equations:

$$
\begin{aligned}
\rho_{n-1}(x, z) & +\Lambda(x) \zeta_{n}(x, z)=0, \\
\rho_{n-1}(x, z) & =-\partial_{x} \zeta^{n-1} F\left(x, \zeta^{n-1}, z\right)+G\left(x, \zeta^{n-1}, z\right), \\
\Lambda(x) & =-\partial_{x} \zeta_{0} \partial_{y} F\left(x, \zeta_{0}, \mu_{0}\right)+\partial_{y} G\left(x, \zeta_{0}, \mu_{0}\right) .
\end{aligned}
$$

Due to our assumption (A1), however, we are potentially dividing by zero as $\Lambda\left(x_{0}\right)=0$ when solving for the updates $\zeta_{n}$. The idea of [5] is then to proceed by removing the singularity in the expression for $\zeta_{n}$ by solving the equation $\rho_{n-1}\left(x_{0}, z\right)=0$ for $z=\mu^{n}$ so that $-\rho_{n-1}\left(x, \mu^{n}\right) / \Lambda(x)$ is well-defined. We collect this into an algorithm in the following:

Algorithm 1 Suppose (A1) and (A2). To compute the canard explosion point do the following:

$1^{\circ}$ Define $\tilde{\Lambda}=\tilde{\Lambda}(x)$ to be

$$
\tilde{\Lambda}(x)=\int_{0}^{1} \partial_{x} \Lambda\left(x_{0}+s\left(x-x_{0}\right), \mu_{0}\right) d s
$$

Then by construction $\Lambda(x)=\left(x-x_{0}\right) \tilde{\Lambda}(x)$ where $\Lambda$ is defined in (24).

$2^{\circ}$ Define

$$
\left.\tilde{e}_{0}(x)=\int_{0}^{1} \partial_{x} \rho_{0}\left(x_{0}+s\left(x-x_{0}\right), \mu_{0}\right)\right) d s .
$$

Then by construction $\rho_{0}\left(x, \mu_{0}\right)=\left(x-x_{0}\right) \tilde{e}_{0}(x)$ where $\rho_{0}$ is defined in (23) $)_{n=1}$.

$2^{\circ}$ Iterate the following over $n$ starting from $n=1$ and $\zeta^{0}=\zeta_{0}$ and $\mu^{0}=\mu_{0}$ until $\left|\tilde{e}_{n}\right|$ has reached a desired tolerance:

(i) Define

$$
\zeta_{n}(x)=-\frac{\tilde{e}_{n-1}(x)}{\tilde{\Lambda}(x)}, \quad \zeta^{n}(x)=\zeta^{n-1}(x)+\zeta_{n}(x)
$$


(ii) Solve the following equation for $z=\mu_{n}$ :

$$
\rho_{n}\left(x_{0}, \mu^{n-1}+z\right)=0,
$$

where

$$
\rho_{n}\left(x, \mu^{n-1}+z\right)=-\partial_{x} \zeta^{n} F\left(x, \zeta^{n}, \mu^{n-1}+z\right)+G\left(x, \zeta^{n}, \mu^{n-1}+z\right) .
$$

(iii) Set

$$
\mu^{n}=\mu^{n-1}+\mu_{n}
$$

and let

$$
\left.\tilde{e}_{n}(x)=\int_{0}^{1} \partial_{x} \rho_{n}\left(x_{0}+s\left(x-x_{0}\right), \mu^{n}\right)\right) d s .
$$

Then by construction $\rho_{n}\left(x, \mu^{n}\right)=\left(x-x_{0}\right) \tilde{e}_{n}(x)$.

The graph $y=\zeta^{n}(x)$ is then the approximation of the canard slow manifold, connecting repelling and attracting branches, at the explosion point $z=\mu^{n}$. The error is described in Theorem 1.

Main result. Our main result is contained in the following theorem which we prove in section 4 .

Theorem 1 Suppose that the assumptions (A1) and (A2) hold true and that $F$ and $G$ are analytic in their arguments $(x, y, z)$. (i) Fix first $n \geq 0$. Then, provided $\epsilon$ is sufficiently small, the procedure defined in Algorithm 1 generates a sequence of $\zeta_{i}$ 's and $\mu_{i}$ 's so that

$$
\zeta^{n}(x)=\sum_{i=0}^{n} \zeta_{i}(x), \quad \mu=\mu^{n}=\sum_{i=0}^{n} \mu_{i}
$$

satisfies (8) up to an error (25) of $\tilde{e}_{n}=\mathcal{O}\left(\epsilon^{n+1}\right)$. Moreover, (ii) there exists an $N(\epsilon)=\mathcal{O}\left(\epsilon^{-1 / 2}\right) \in$ $\mathbb{N}$ so that the error in (25) with $n=N$ is exponentially small $\tilde{e}_{N}=\mathcal{O}\left(e^{-c / \epsilon^{1 / 2}}\right)$, with $c>0$ and independent of $\epsilon$.

The first part (i) only requires smoothness. The last part (ii) requires analyticity.

Remark 6 The $\sqrt{\epsilon}$ in Theorem 1 is in agreement with the results in $[6,10]$ where the canard point is obtained as a smooth function of $\sqrt{\epsilon}$.

\section{Applications}

\subsection{Van der Pol}

In this section we consider the classical van der Pol system

$$
\begin{aligned}
& \dot{x}=F(x, y)=y-\frac{1}{3} x^{3}+x, \\
& \dot{y}=G(x, z)=\epsilon(z-x) .
\end{aligned}
$$

This system has a canard explosion near $z=\mu_{0}=1$ for $\epsilon$ small. Asymptotic expansions yield a more accurate value

$$
\mu=1-\frac{1}{8} \epsilon-\frac{3}{32} \epsilon^{2}-\frac{173}{1024} \epsilon^{3}+\mathcal{O}\left(\epsilon^{4}\right)
$$


see e.g. [1, 17]. To use Algorithm 1 to compute the canard explosion point we first verify the conditions (A1) and (A2). Solving $F(x, y)$ for $y$ gives

$$
y=\zeta_{0}(x)=\frac{1}{3} x^{3}-x .
$$

Then

$$
\Lambda(x)=-\partial_{x} \zeta_{0} \partial_{y} F\left(x, \zeta_{0}(x)\right)=1-x^{2} .
$$

We have a fold point at $x=x_{0}=1$ where $\partial_{x} \zeta_{0}\left(x_{0}\right)=0$ where also $\Lambda$ vanishes. To complete the verification of (A1) we must solve $G\left(x_{0}, z\right)=0$ for $z=\mu_{0}$. We obtain $\mu_{0}=1$. For (A2) note that

$$
\tilde{\Lambda}(x)=-(1+x)
$$

dividing $x-x_{0}=x-1$ out, so that $\tilde{\Lambda}\left(x_{0}\right)=-2 \neq 0$. Since $\Phi \equiv \epsilon$ we have to take case (b) in (A2). The remaining assumptions can easily be verified for $\epsilon$ sufficiently small.

We are now ready to apply Algorithm 1 . For $1^{\circ}$ we have (27) and for $2^{\circ}$ we first note that

$$
\rho_{0}(x, z)=\epsilon(z-x)
$$

and so $\tilde{e}_{0}=-\epsilon$ when dividing $x-x_{0}=x-1$ out from $e_{0}(x)=\rho_{0}\left(x, \mu_{0}\right)$.

For $3^{\circ}$ we first set $\zeta_{1}=-\tilde{e}_{0} / \tilde{\Lambda}=-\epsilon /(1+x)$, which finishes step (i) and define $\rho_{1}$ (ii) as

$$
\rho_{1}(x, z)=\epsilon(1+z)-\epsilon(1+x)^{-3}\left(x^{3}+3 x^{2}+3 x+1-\epsilon\right) .
$$

Setting $\rho_{1}\left(x_{0}, z\right)=0$ gives $z=\mu_{1}=-\frac{1}{8} \epsilon$ so that

$$
\mu^{1}=1+\mu_{1}=1-\frac{1}{8} \epsilon,
$$

correct to first order in $\epsilon$ cf. (26). This finishes step (iii). We iterate this procedure and obtain the following approximations

$$
\mu^{2}=1-\frac{1}{8} \epsilon+\frac{3}{32} \epsilon^{2}-\frac{27}{2048} \epsilon^{3}, \mu^{3}=1-\frac{1}{8} \epsilon+\frac{3}{32} \epsilon^{2}-\frac{173}{1024} \epsilon^{3}+\mathcal{O}\left(\epsilon^{4}\right),
$$

correct to order 2 respectively 3 in $\epsilon$.

\subsection{Templator}

In this section we consider the Templator model $[2,4]$

$$
\begin{aligned}
& \dot{x}=F(x, y)=k_{u} y^{2}+k_{T} y^{2} x-\frac{q x}{K+x}, \\
& \dot{y}=G(x, y, z)=z-k_{u} y^{2}-k_{T} y^{2} x
\end{aligned}
$$

and use Algorithm 1 to compute a canard explosion. Numerical computations indicate a canard explosion at

$$
\mu=0.419943,
$$

$[2,4]$. This system has no explicit $\epsilon$, yet numerical simulations show that the system exhibits a slow-fast structure. In [4] it is shown that various combinations of the parameters in the system 
can locally be considered as small parameters, but no global parametrization in the form (2) exists. Here we proceed to find a canard explosion without any identification of an explicit small parameter. As in [4] we set $k_{u}=0.01, k_{T}=1, q=1$, and $K=0.05$. We first solve $F(x, y)=0$ for $y=\zeta_{0}(x)$ and obtain

$$
\zeta_{0}(x)=\frac{50 \sqrt{2 x\left(1+150 x+5000 x^{2}\right)}}{1+150 x+5000 x^{2}} .
$$

Note that (32) is independent of $z$. The equation also has a negative solution which we have discarded. We then realize that there is a point $x=\sqrt{2} / 100=0.014142$ where $\partial_{x} \zeta_{0}=0$. The function $\Lambda(x)=-\partial_{x} \zeta_{0} \partial_{y} F\left(x, \zeta_{0}\right)+\partial_{y} G\left(x, \zeta_{0}, z\right)$ vanishes near this point at $x=x_{0}=0.014345$. For this value of $x=x_{0}$, we continue to verify the assumptions in (A1), and compute $z=\mu_{0}$ giving $G\left(x_{0}, \zeta_{0}\left(x_{0}\right), z\right)=0$. We obtain $z=\mu_{0}=0.417681$. The error is $0.5 \%$ in comparison with the value in (31). We then define $\tilde{\Lambda}$ and $\tilde{e}_{0}$ by division of $\Lambda$ and $e_{0}$ by $x-x_{0}$. To verify (A2) we note that

$$
\begin{aligned}
\Phi & \equiv 1, \\
\tilde{\Lambda}\left(x_{0}\right) & =996.78, \\
\tilde{e}_{0}\left(x_{0}\right) & =-17.157,
\end{aligned}
$$

so that

$$
\frac{\tilde{e}_{0}\left(x_{0}\right)}{\tilde{\Lambda}\left(x_{0}\right)}=0.017213
$$

Since (33) is "small", we are confident that (A2), case (a), is satisfied and we therefore proceed by applying Algorithm 1. Introducing $\zeta_{1}(x)=-\tilde{e}_{0}(x) / \tilde{\Lambda}(x)$ then gives the new error

$$
\rho_{1}(x, z)=-\partial_{x} \zeta^{1} F\left(x, \zeta^{1}\right)+G\left(x, \zeta^{1}, \mu_{0}+z\right), \quad \zeta^{1}=\zeta_{0}+\zeta_{1} .
$$

We solve $\rho_{1}\left(x_{0}, z\right)=0$ for $z=\mu_{1}$ and obtain the improved approximation to the canard explosion point

$$
\mu^{1}=\mu_{0}+\mu_{1}=0.419883
$$

an error of $0.01 \%$. At the next step we get $\mu^{2}=0.419938$. The error is now $0.001 \%$.

\subsection{Rotated van der Pol}

In this section we again consider the van der Pol equations, but we rotate the coordinates, replacing $x$ by $x-y$ and $y$ by $x+y$ :

$$
\begin{aligned}
& \dot{x}=F(x, y, z)=\frac{1}{2}\left(2 x-(x-y)^{3} / 3+\frac{1}{2} \epsilon(\mu-(x-y)),\right. \\
& \dot{y}=G(x, y, z)=-\frac{1}{2}\left(2 x-(x-y)^{3} / 3\right)+\frac{1}{2} \epsilon(\mu-(x-y)) .
\end{aligned}
$$

mimicking a situation where the slow and fast variables have not been properly identified. We will demonstrate that the algorithm applies to this case too. The fold point in the original variables is no longer a fold point in the sense used in section 3.1 in the coordinates used here. Indeed solving for the $x$-nullcline gives

$$
y=\zeta_{0}(x)=x-6^{1 / 3} x^{1 / 3}+\mathcal{O}(\epsilon)
$$


with non-zero derivative for the relevant $x$-values. The fold point now appears where $\Lambda(x)=$ $1-6^{2 / 3} x^{2 / 3}+\epsilon 6^{-2 / 3} x^{-2 / 3}$ vanishes. The $\mathcal{O}(\epsilon)$-term in $\Lambda$ is following the discussion in Remark 4 not important: It can collected into $a_{0}=\mathcal{O}(\epsilon)$ in (11). We therefore re-define $\Lambda$ as $\Lambda(x)=1-6^{2 / 3} x^{2 / 3}$. Then $\Lambda$ vanishes at the point $x=1 / 6$, where $y=-5 / 6+\mathcal{O}(\epsilon)$ according to (34). The point $(x, y)=(1 / 6,-5 / 6)$ is also the value obtained by transforming the fold point point $(1,-2 / 3)$ in the original variables, used in section 3.1, to the current rotated variables. This alteration of $\Lambda$ is in principle not needed: There is a point $\epsilon$-close to the point above where the old $\Lambda$ vanishes. However, when we ignore this part then the calculations can actually be done by hand without using a computer algebra software.

Note that $\partial_{x} \Lambda\left(x_{0}\right)=-4 \neq 0$ and we can therefore define

$$
\tilde{\Lambda}(x)=-4+4\left(x-x_{0}\right)-\frac{32}{3}\left(x-x_{0}\right)^{2}+\mathcal{O}\left(\left(x-x_{0}\right)^{3}\right) .
$$

Now we are in a position to apply Algorithm $12^{\circ}$. For this we first note that

$$
\rho_{0}(x, z)=-\partial_{x} \zeta_{0} F\left(x, \zeta_{0}\right)+G\left(x, \zeta_{0}, z\right)=\epsilon 6^{-2 / 3} x^{-2 / 3}\left(z-6^{1 / 3} x^{1 / 3}\right),
$$

and we therefore obtain $z=\mu_{0}=1$ by solving $\rho_{0}\left(x_{0}, z\right)=0$. Following (iii), we then set

$$
\tilde{e}_{0}(x)=-\frac{2}{3} \epsilon\left(3-18\left(x-x_{0}\right)+104\left(x-x_{0}\right)^{2}+\mathcal{O}\left(\left(x-x_{0}\right)^{3}\right) .\right.
$$

It is easy to verify the conditions in (A2). We therefore proceed as in Algorithm $1\left(3^{\circ}\right)$ starting from (i) setting $\zeta_{1}(x)=-e_{0}(x) / \Lambda(x)=-\epsilon 6^{-2 / 3} /\left(6^{1 / 3} x^{1 / 3}+1\right) x^{2 / 3}$ which by construction is smooth also at $x=x_{0}$. We continue and obtain

$$
\begin{aligned}
& \mu^{1}=\mu_{0}+\mu_{1}=1-\frac{1}{8} \epsilon-\frac{35}{32} \epsilon^{2}-\frac{545}{384} \epsilon^{3}+\mathcal{O}\left(\epsilon^{4}\right), \\
& \mu^{2}=\mu_{0}+\mu_{1}+\mu_{2}=1-\frac{1}{8} \epsilon-\frac{3}{32} \epsilon^{2}-\frac{18183}{2048} \epsilon^{3}+\mathcal{O}\left(\epsilon^{4}\right), \\
& \mu^{3}=\mu_{0}+\mu_{1}+\mu_{2}+\mu_{3}=1-\frac{1}{8} \epsilon+\frac{3}{32} \epsilon^{2}-\frac{173}{1024} \epsilon^{3}+\mathcal{O}\left(\epsilon^{4}\right)
\end{aligned}
$$

correct to order 1,2 respectively $3 \mathrm{cf}$. (26). Note that the corrections in $\mu^{1}$ (the terms $-\frac{35}{32} \epsilon^{2}-$ $\frac{545}{384} \epsilon^{3}$ ) and $\mu^{2}$ (the term $-\frac{18183}{2048} \epsilon^{3}$ ) are different from those in (28) and (29) being 0 and $-\frac{27}{2048} \epsilon^{3}$, respectively, but the expressions are correct to the order expected by Theorem 1, even though the slow and fast variables have not been properly identified.

\subsection{Templator again}

As a final example we consider the Templator model (30) again with the parameters $k_{u}=0.01$, $k_{T}=1, q=1$, and $K=0.05$ as before. The solution of $F(x, y)=0$ gave

$$
y=\zeta_{0}(x)=\frac{50 \sqrt{2 x\left(1+150 x+5000 x^{2}\right)}}{1+150 x+5000 x^{2}},
$$

having one single fold point at $x=x_{0}=\sqrt{2} / 100$. There is, however, another canard explosion at

$$
\mu=0.967555
$$

[2] that arises from another zero of $\Lambda$. Here we cannot ignore the term $\partial_{y} G$ in $\Lambda$ as we did in the rotated van der Pol above. This term is not small as is illustrated in Fig. 1. Here it is made 
visible that $\Lambda$ has another zero at $x_{0}=0.599393$ where $\partial_{x} \Lambda<0$. Therefore we can define $\tilde{\Lambda}$ by $\Lambda(x)=\left(x-x_{0}\right) \tilde{\Lambda}(x)$. The zero of $\Lambda$ gives rise to a singularity in the Eq. (22). To continue the verification of (A1) we note that solving $\rho_{0}\left(x_{0}, z\right)=0$ gives

$$
\mu_{0}=0.967710 \text {. }
$$

This gives a relative error of $0.01 \%$ in comparison with the value in (35). To verify the conditions (A2) we note the following:

$$
\begin{aligned}
\tilde{\Lambda}\left(x_{0}\right) & =-3.6535, \\
\tilde{e}_{0}\left(x_{0}\right) & =-0.0521311,
\end{aligned}
$$

and $\Phi \equiv 1$ as above, so that

$$
\frac{\tilde{e}_{0}\left(x_{0}\right)}{\tilde{\Lambda}\left(x_{0}\right)}=0.014268
$$

This again gives us confidence to apply Algorithm 1. The expressions are quite messy so we leave out the details and just present the result of one iteration of $2^{\circ}$ of the method:

$$
\mu^{1}=0.967560
$$

The error is now $0.006 \%$. A final additional application gives

$$
\mu^{2}=0.967558
$$

reducing the relative error to $3 \times 10^{-6}$.

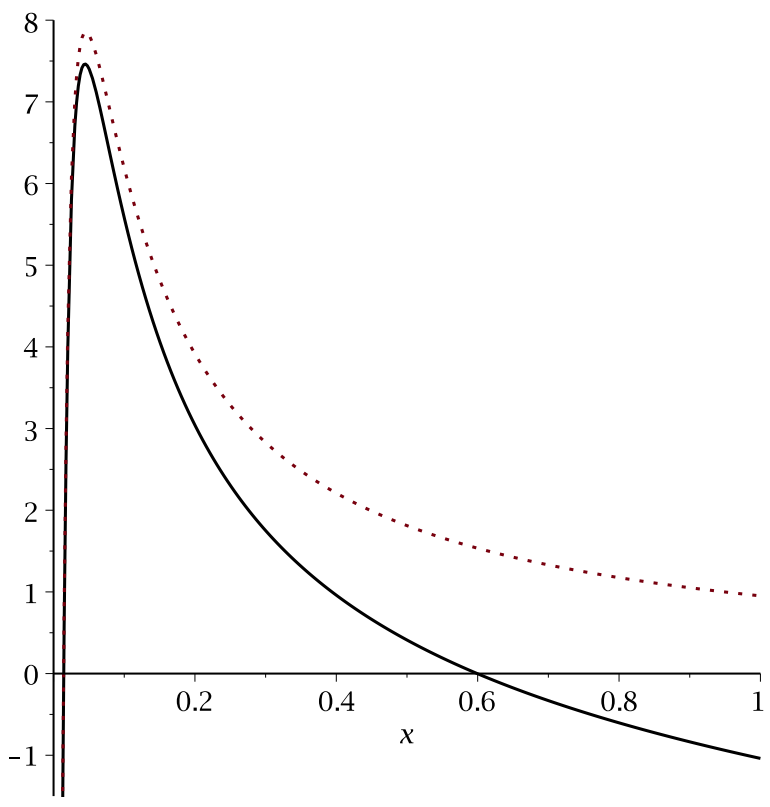

Figure 1: The graph of $\Lambda$ (14) and $\Lambda-\partial_{y} G=-\partial_{x} \zeta_{0} \partial_{y} F$ (dotted). 


\section{Proof of Theorem 1}

To prove our theorem we start from (11):

$$
\begin{aligned}
\dot{x} & =F_{0}\left(x, y_{0}, z_{0}\right)=f_{0}(x)+F_{0 y}\left(x, y_{0}, z_{0}\right) y_{0}+F_{0 z}\left(x, y_{0}, z_{0}\right) z_{0}, \\
\dot{y}_{0} & =e_{0}(x)+\left(\Phi(x)+b_{0}(x)\right) z_{0}+T_{0}\left(x, z_{0}\right)+\left(\Lambda(x)+a_{0}\left(x, z_{0}\right)\right) y_{0}+R_{0}\left(x, y_{0}, z_{0}\right), \\
\dot{z}_{0} & =0
\end{aligned}
$$

where $f_{0} \equiv 0$ and $b_{0} \equiv 0$. From conditions (A1) and (A2), it is without loss of generality to take $K \equiv\left|\tilde{\Lambda}^{-1}\right|_{\nu_{0}}=1$. Indeed, we can introduce a new time $\tau=K^{-1} t$ to achieve this. Then also by (A2):

$$
\tilde{\delta}_{0} \equiv|\tilde{e}|_{\nu_{0}} \leq \epsilon \ll 1
$$

and furthermore, assuming case (a):

$$
\left|\Phi\left(x_{0}\right)\right|^{-1},|\Phi| \leq C_{\Phi}
$$

Here $C_{\Phi}>0$ is independent of $\epsilon$. The proof for case (b) where

$$
\left|\Phi\left(x_{0}\right)\right|^{-1} \leq C_{\Phi} / \epsilon,|\Phi| \leq C_{\Phi} \epsilon,
$$

is almost identical (see Remark 7 below).

We will seek to apply a sequence of transformations $\phi_{1}, \phi_{2}, \ldots, \phi_{n}$ to (11) that successively seek to diminish the $\tilde{e}_{i}$ 's that appear as a result of these transformations. We define the transformations through an iterative lemma.

The iterative lemma. To set up an iterative lemma we start from the normal form (36) with appropriate subscripts removed:

$$
\begin{aligned}
& \dot{x}=F(x, y, z)=f(x)+F_{y}(x, y, z) y+F_{z}(x, y, z) z, \\
& \dot{y}=e(x)+(\Phi(x)+b(x)) z+T(x, z)+(\Lambda(x)+a(x, z)) y+R(x, y, z), \\
& \dot{z}=0,
\end{aligned}
$$

where

$$
e=\left(x-x_{0}\right) \tilde{e}(x)
$$

$T=\mathcal{O}\left(z^{2}\right)$ independent of $y$, and $R=\mathcal{O}\left(y^{2}\right)$. We suppose

$$
\tilde{\delta}=|\tilde{e}|_{\nu},|a(x, 0)|_{\nu},\left|\epsilon^{-1} b\left(x_{0}\right)\right| \leq c \epsilon,
$$

for some constant $c>0$ and $\epsilon$ sufficiently small. Note that these conditions are satisfied for our initial system (36).

Then $y=0, z=0$ is an approximation to the canard solution. The accuracy of the approximation is determined by $\tilde{\delta}$. We will in the following apply a transformation $\phi$ to (40), which will be based on two steps, seeking to improve the approximation of the canard solution. First, we define the solution, $\zeta=\zeta(x)$, of the equation

$$
e(x)+\Lambda(x) \zeta(x)=0 .
$$

The solution is

$$
\zeta(x)=\frac{\tilde{e}(x)}{\tilde{\Lambda}(x)},
$$


measuring

$$
|\zeta|_{\nu} \leq K \tilde{\delta}
$$

Now, we set

$$
y=\zeta(x)+y_{+}
$$

This gives

$$
\begin{aligned}
\dot{x} & =f^{+}(x)+F_{y}^{+}\left(x, y_{+}, z\right) y_{+}+F_{z}^{+}\left(x, y_{+}, z\right) z, \\
\dot{y}_{+} & =e^{+}(x)+\left(\Phi(x)+b^{+}(x)\right) z+T^{+}(x, z)+\left(\Lambda(x)+a^{+}(x, z)\right) y+R^{+}(x, y, z), \\
\dot{z} & =0
\end{aligned}
$$

with

$$
\begin{aligned}
f^{+}(x) & =f(x)+F_{y}(x, \zeta, 0) \zeta, \\
F_{z}^{+}\left(x, y_{+}, z\right) & =F_{z}\left(x, \zeta+y_{+}, z\right), \\
e^{+}(x) & =-\partial_{x} \zeta f^{+}(x)+a(x, 0) \zeta+R_{+}(x, \zeta, 0), \\
b^{+}(x) & =b(x)-\partial_{x} \zeta F_{z}(x, \zeta, 0)+\partial_{z} a(x, 0) \zeta+\partial_{z} R_{+}(x, \zeta, 0), \\
a^{+}(x, z) & =a(x, z)-\partial_{x} \zeta F_{y}(x, \zeta, 0)+\partial_{y} R_{+}(x, \zeta, 0),
\end{aligned}
$$

and where $F_{y}^{+}, T^{+}=\mathcal{O}\left(z^{2}\right)$, which is independent of $y$, and $R^{+}=\mathcal{O}\left(y^{2}\right)$ are determined by Taylor's theorem. For $\epsilon$ sufficiently small we then have

$$
\delta^{+} \equiv\left|e^{+}\right|_{\nu-\xi} \leq \frac{\epsilon C \tilde{\delta}}{\xi},
$$

for $C$ sufficiently large, using (41), (44) and the fact that $R^{+}(x, y, z)=\mathcal{O}\left(y^{2}\right)$ is quadratic.

The result is not yet appropriate for iteration as

$$
e^{+}\left(x_{0}\right) \neq 0 \text {. }
$$

To account for this we transform $z$ by introducing $z=\mu+z_{+}$with $\mu$ satisfying

$$
e^{+}\left(x_{0}\right)+\left(\Phi\left(x_{0}\right)+b^{+}\left(x_{0}\right)\right) \mu+T^{+}\left(x_{0}, \mu\right)=0 .
$$

By (38), and the contraction mapping theorem, there exists a solution

$$
\mu \approx-\frac{e^{+}\left(x_{0}\right)}{\Phi\left(x_{0}\right)}
$$

of (45), that satisfies

$$
|\mu| \leq 2 C_{\Phi} \delta^{+} \leq \frac{2 \epsilon C_{\Phi} C \tilde{\delta}}{\xi}
$$

Here we have used that $T^{+}=\mathcal{O}\left(z^{2}\right),(41)$, the first estimate in (38) and the smallness of $\tilde{\delta}$ and $\epsilon$. Then the resulting system reads

$$
\begin{aligned}
\dot{x} & =F\left(x, \zeta+y_{+}, \mu+z_{+}\right)=f_{+}(x)+F_{+y}\left(x, y_{+}, z_{+}\right) y_{+}+F_{+z}\left(x, y_{+}, z_{+}\right) z_{+}, \\
\dot{y}_{+} & =e_{+}(x)+\left(\Phi(x)+b_{+}(x)\right) z_{+}+T_{+}\left(x, z_{+}\right)+\left(\Lambda(x)+a_{+}\left(x, z_{+}\right)\right) y+R_{+}\left(x, y, z_{+}\right), \\
\dot{z}_{+} & =0
\end{aligned}
$$


with

$$
\begin{aligned}
f_{+}(x) & =f^{+}(x)+F_{z}^{+}(x, 0, \mu) \mu, \\
F_{+y}\left(x, y_{+}, z_{+}\right) & =F_{y}^{+}\left(x, y_{+}, \mu+z_{+}\right) \\
e_{+}(x) & =e^{+}(x)+\left(\Phi(x)+b^{+}(x)\right) \mu+T^{+}(x, \mu), \\
b_{+}(x) & =b^{+}(x)+\partial_{z} T^{+}(x, \mu),
\end{aligned}
$$

and where $F_{+z}, T_{+}, R_{+}$are determined by Taylor's theorem. By construction $e_{+}\left(x_{0}\right)=0$. We estimate the new obstacle to invariance $e_{+}$of $y_{+}=0, z_{+}=0$ as

$$
\begin{aligned}
\left|e_{+}\right|_{\nu-\xi} & \leq\left|e^{+}\right|_{\nu-\xi}+2 C_{\Phi}|\mu| \\
& \leq \frac{\epsilon C\left(1+2 C_{\Phi}^{2}\right) \tilde{\delta}}{\xi},
\end{aligned}
$$

using the second estimate in (38) and (41), for $\tilde{\delta}$ and $\epsilon$ sufficiently small. Since $e_{+}\left(x_{0}\right)=0$ we write $e_{+}$as

$$
e_{+}(x)=\left(x-x_{0}\right) \tilde{e}_{+}(x), \quad \tilde{e}_{+}(x)=\int_{0}^{1} \partial_{x} e_{+}\left(x_{0}+s\left(x-x_{0}\right)\right) d s
$$

and estimate our new error $\tilde{e}_{+}$on $\nu_{+}=\nu-2 \xi$ using a Cauchy estimate and (48):

$$
\left|\tilde{e}_{+}\right|_{\nu_{+}} \leq \frac{\epsilon C\left(1+2 C_{\Phi}^{2}\right) \tilde{\delta}}{\xi^{2}} .
$$

Remark 7 If we assume case (b) in (A2) then we obtain a $\epsilon^{-1}$-factor in (46) so that $|\mu| \leq$ $2 \epsilon^{-1}\left|\Phi\left(x_{0}\right)\right|^{-1} \delta^{+}$. However, we recover an estimate as in (48) using (39) (in place of (38) used above).

We collect the results in the following iterative lemma:

Lemma 3 Let $\xi>0$ and $\nu_{+} \equiv \nu-2 \xi \geq 0$. Then there exists a $\bar{C}>0$ so that the transformation

$$
\begin{aligned}
\phi:(x, y, z) & \mapsto\left(x_{+}, y_{+}, z_{+}\right), \\
x_{+} & =x \\
y_{+} & =\zeta(x)-y, \\
z_{+} & =\mu-z,
\end{aligned}
$$

with $\zeta$ and $\mu$ solving (43) and (45), respectively, maps (40) into (47) where

$$
\left|a-a_{+}\right|_{\nu_{+}},\left|b-b_{+}\right|_{\nu_{+}},\left|R-R_{+}\right|_{\nu_{+}},\left|T-T_{+}\right|_{\nu_{+}} \leq \bar{C} \tilde{\delta},
$$

and

$$
\tilde{\delta}_{+} \equiv\left|\tilde{e}_{+}\right|_{\nu_{+}} \leq \frac{\epsilon \bar{C}}{\xi^{2}} \tilde{\delta},
$$

provided $\epsilon$ are sufficiently small. 
The $\mathcal{O}\left(\epsilon^{n}\right)$ estimates in (i) of Theorem 1 follow directly from (49) as each application of the procedure introduces a factor of $\epsilon$.

Exponential estimates. To obtain the exponential estimates we first apply the iterative lemma, Lemma 3, to (36) and obtain

$$
\tilde{\delta}_{1}=\left|\tilde{e}_{1}\right|_{\nu_{1}} \leq \frac{\epsilon \bar{C}_{0}}{\xi_{0}^{2}} \tilde{\delta}_{0}=\frac{16 \bar{C}_{0}}{\nu_{0}^{2}} \epsilon^{2}
$$

using (37) and (49) with $\nu_{1}=\frac{\nu_{0}}{2}$ setting here $\xi=\xi_{0}=\frac{\nu_{0}}{4}=\mathcal{O}(1) \cdot{ }^{1}$ Here $C_{0}$ is the constant obtained from applying Lemma 3 to (36). Then we apply the lemma successively setting the measure of the domain reduction $\xi$ in Lemma 3 to be $^{2}$

$$
\xi=\sqrt{2 \epsilon \bar{C}_{\infty}}
$$

with

$$
\bar{C}_{\infty}=2 \bar{C}_{0}
$$

The $(n+1)$ th application of Lemma 3 gives rise to a constant $\bar{C}_{n}$. Then by $(49)$

$$
\tilde{\delta}_{n+1} \leq \frac{\epsilon \bar{C}_{n+1}}{\xi_{n}^{2}} \tilde{\delta}_{n} \leq 2^{-1} \tilde{\delta}_{n-1} \leq 2^{-n} \tilde{\delta}_{1},
$$

while $\bar{C}_{n+1} \leq \bar{C}_{\infty}=2 \bar{C}_{0}$ and

$$
\nu_{n+1}=\nu_{n}-2 \xi=\frac{1}{2} \nu_{0}-2 n \xi \geq 0
$$

By the geometric sum formula and the fact that $\tilde{\delta}_{1}=\mathcal{O}\left(\epsilon^{2}\right)$, cf. (50), the requirement $\bar{C}_{n} \leq 2 \bar{C}_{0}$ does not pose any restrictions on $n$ for $\epsilon$ sufficiently small. The only restriction on $n$ is contained in the last inequality in (53):

$$
n \leq N(\epsilon) \equiv\left\lfloor\frac{\nu_{0}}{4 \xi}\right\rfloor=\left\lfloor\frac{\nu_{0}}{4 \sqrt{2 \epsilon \bar{C}_{\infty}}}\right\rfloor .
$$

Here $\lfloor v\rfloor$ denotes the integer part of $v \geq 0$. Hence Lemma 3 can be applied $\mathcal{O}\left(\epsilon^{1 / 2}\right)$-many times, which by (52) with $n=N(\epsilon)$ gives the exponential estimate

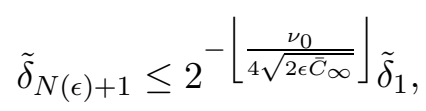

in Theorem 1. This then completes the proof.

\section{References}

[1] E. Benoit, J. L. Callot, F. Diener, and M. Diener. Chasse au canard. Collectanea Mathematica, 32:37-119, 1981.

\footnotetext{
${ }^{1}$ We could in principle take any $\xi=\mathcal{O}(1)$ satisfying $\xi<\frac{\nu_{0}}{2}$

${ }^{2}$ In fact, any $\xi=C^{-1} \sqrt{\epsilon}$ for $C$ sufficiently large would do.
} 
[2] K. M. Beutel and E. Peacock-López. Complex dynamics in a cross-catalytic self-replication mechanisms. Journal of Chemical Physics, 126(125104), 2007.

[3] M. Brøns. Relaxation oscillations and canards in a nonlinear model of discontinuous plastic deformation in metals at very low temperatures. Proceedings of the Royal Society of London. Series A: Mathematical, Physical and Engineering Sciences, 461:2289-2302, 2005.

[4] M. Brøns. Canard explosion of limit cycles in templator models of self-replication mechanisms. Journal of Chemical Physics, 134(144105), 2011.

[5] M. Brøns. An iterative method for the canard explosion in general planar systems. Discrete and Continuous Dynamical Systems Supplement 2013, pages 77-83, 2013.

[6] F. Dumortier and R. Roussarie. Canard cycles and center manifolds. Memoirs of the American Mathematical Society, 1996.

[7] Neil Fenichel. Geometric singular perturbation theory for ordinary differential equations. Journal of Differential Equations, 31(1):53-98, 1979.

[8] S.J. Fraser. The steady state and equilibrium approximations: a geometrical picture. Journal of Chemical Physics, 88:4732-4738, 1990.

[9] C. K. R. T. Jones. Geometric singular perturbation theory. Lecture Notes in Mathematics, 1609:44-118, 1995.

[10] M. Krupa and P. Szmolyan. Extending geometric singular perturbation theory to nonhyperbolic points - fold and canard points in two dimensions. SIAM Journal of Mathematical Analysis, 33:286-314, 2001.

[11] M. Krupa and P. Szmolyan. Relaxation oscillation and canard explosion. Journal of Differential Equations, 174:312-368, 2001.

[12] C. Kuehn. From first Lyapunov coefficients to maximal canards. International Journal of Bifurcation and Chaos, 20(5):1467-1475, 2010.

[13] A. Neishtadt. Persistence of stability loss for dynamical bifurcation, i. Journal of Differential Equations, 23:1385-1390, 1987.

[14] M.R. Roussel and S.J. Fraser. Geometry of the steady-state approxiation: perturbation and accelerated convergence method. Journal of Chemical Physics, 93:1072-1081, 1990.

[15] W. Rudin. Real and Complex Analysis, 3rd Ed. McGraw-Hill, Inc., New York, NY, USA, 1987.

[16] K. Uldall Kristiansen and C. Wulff. Exponential estimates of slow manifolds. arXiv:1208.4219v1 [math.DS], 2012.

[17] A. K. Zvonkin and M. A. Shubin. Non-standard analysis and singular perturbations of ordinary differential equations. Russian Mathematical Surveys, 39(2):69-131, 1984. 